\title{
Fat accumulation in enterocytes: a key to the diagnosis of abetalipoproteinemia or homozygous hypobetalipoproteinemia
}

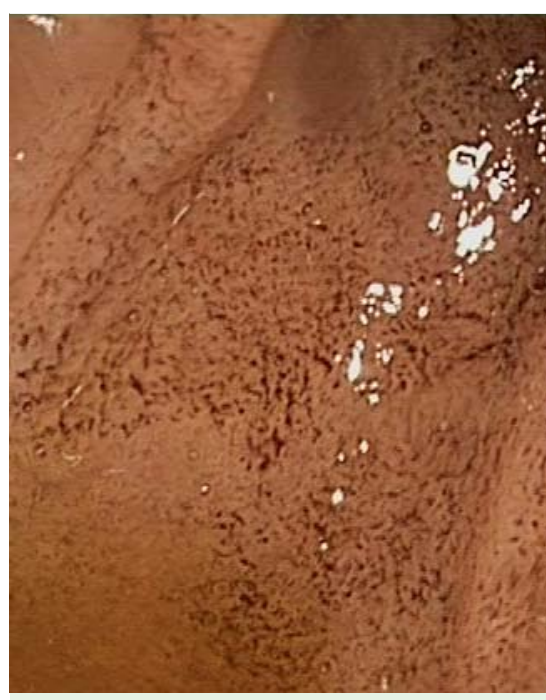

Fig. 1 A 20-year-old woman was referred by her ophthalmologist to investigate the reason for her hypovitaminosis A and secondary night blindness. A macroscopic image taken during gastroscopy shows a pale duodenal mucosa.

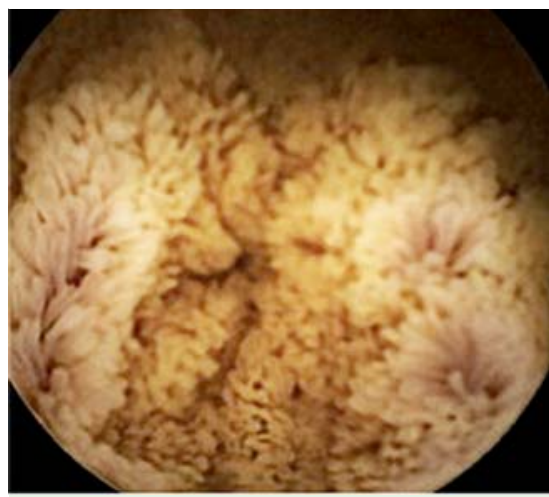

Fig. 2 Videocapsule image illustrating the pale yet pronounced aspect of the villi.

A 20-year-old woman was referred by her ophthalmologist to investigate the reason for her hypovitaminosis A and secondary night blindness. She had no other symptoms of deficiencies in fat-soluble vitamins, no abdominal complaints, and no weight loss.

Laboratory examination revealed a deficiency in vitamin $A(<12 \mu \mathrm{g} / \mathrm{dL}$, normal range $30-80)$ and vitamin $\mathrm{E}(<0.30 \mathrm{mg} / \mathrm{dL}$, normal range $0.5-1.8$ ), a very low prothrombin time (30\%), and very low levels of cholesterol $(30 \mathrm{mg} / \mathrm{dL})$, triglycerides ( $4 \mathrm{mg} / \mathrm{dL}$ ), and LDL-cholesterol (below the

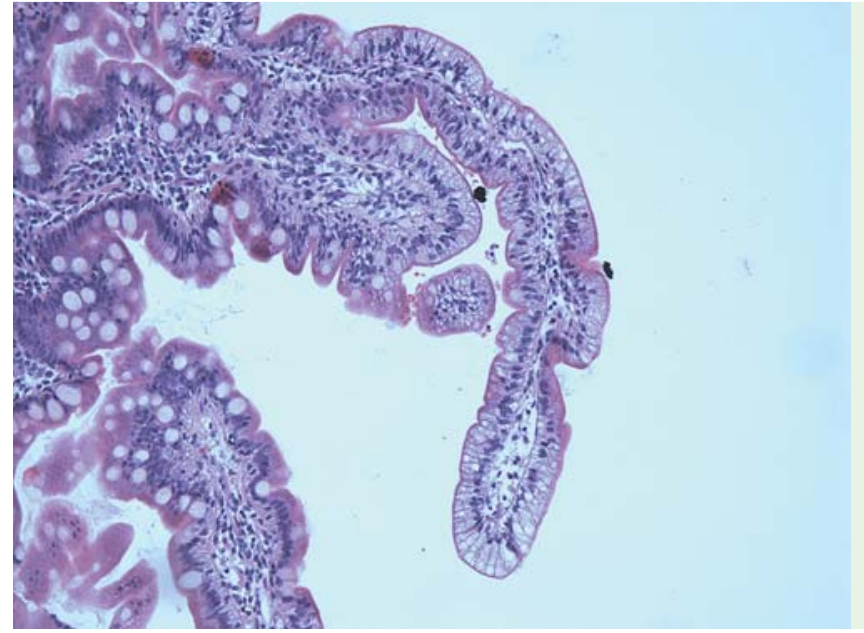

Fig. 3 Microscopic image showing vacuolization, especially on the top of the villi. Vacuolization causes a paler aspect because of fat dissolving during the process of embedding the tissue in paraffin wax ("empty" vacuoles instead of fat accumulation).

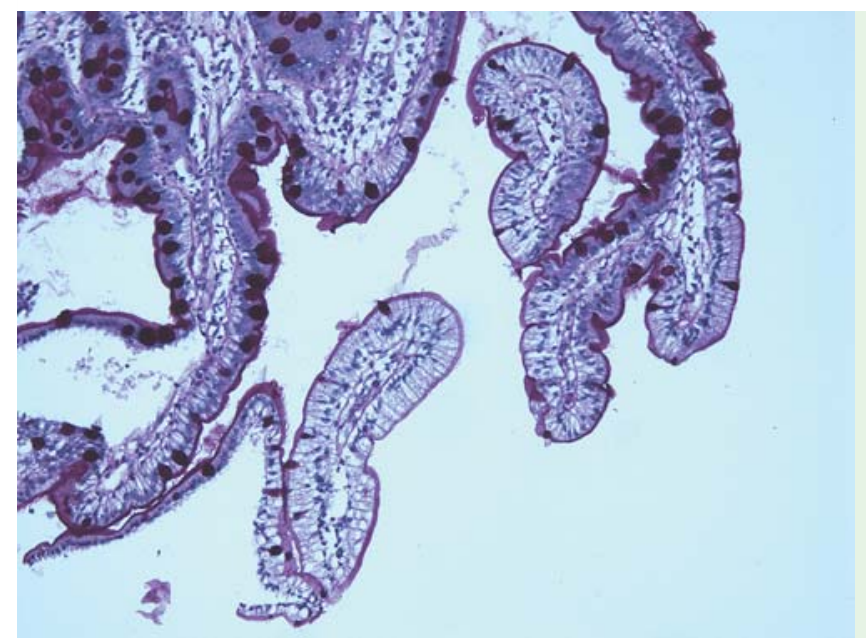

Fig. 4 Negative periodic acid-Schiff staining shows no microorganisms nor accumulation of glycogen, supporting the assumption that the vacuolization is due to lipid accumulation.

level of detection). Her level of 25-hydroxy vitamin $\mathrm{D}$ appeared to be normal, but at the time of her first admission, vitamin D substitution had already been started. A slightly raised alanine aminotransferase was also detected (33U/L).

Further work-up excluded cystic fibrosis, exocrine pancreas insufficiency, and celiac disease. Gastroscopy revealed a very pale duodenal mucosa; the villi, however, could be easily recognized ( $\bullet$ Fig. 1 ). Videocapsule endoscopy revealed a pale small bowel mucosa with extremely pronounced villi ( $\odot$ Fig. 2, $\odot$ Video 1 ). Biopsies of the duodenal mucosa revealed areas of extended supranuclear vacuolization of the cytoplasm in the villi. These areas were interspersed with normal areas ( $\bullet$ Fig. 3 and $\odot$ Fig. 4 ).
These findings suggested a diagnosis of either abetalipoproteinemia or homozygous hypolipobetaproteinemia, disorders that are caused by mutations in both alleles of the microsomal triglycerides transfer protein (MTP) or in the APO-B gene, respectively $[1-2]$ This results in the failure of APO B-100 synthesis in the liver and APO B-48 synthesis in enterocytes, leading to fat accumulation in the small intestine. This diagnosis can be con-

\section{Video 1}

Videocapsule endoscopy in a 20-year-old woman suffering from hypovitaminosis $A$ and secondary night blindness revealed a pale small-bowel mucosa with extremely pronounced villi. 
firmed by sequencing the MTP and APO-B genes [1].

This disorder can be treated by a low-fat diet, supplementation of essential fatty acids, and high oral doses of fat-soluble vitamins [1]. Follow-up is necessary to monitor potential ophthalmologic, neurologic, hematologic, and hepatologic complications [1-2].

This patient illustrates that the disorder is sometimes diagnosed in adulthood when the phenotype is mild [1-3]. The prognosis is variable but adherence to the treatment regime can restore neurological function and prevent subsequent disease progression [1-2].

Endoscopy_UCTN_Code_CCL_1AB_2AZ_3AZ

Competing interests: None

\section{Lobke Desomer, Martine De Vos, Danny De Looze}

Department of Gastroenterology and Hepatology, Ghent University Hospital, Gent, Belgium

\section{Acknowledgment}

\section{$\nabla$}

Histologic images were kindly provided by Stephanie Verschuere, MD, Pathology Department UZ Ghent.

\section{References}

1 Lee $J$, Hegele R. Abetalipoproteinemia and homozygous hypobetalipoproteinemia: a framework for diagnosis and management. J Inherit Metab Dis 2014; 37: 333 - 339

2 Cefalù A, Pirruccello J, Noto $D$ et al. A Novel $A P O B$ mutation identified by exome sequencing cosegregates with steatosis, liver cancer, and hypocholesterolemia.
Arterioscler Thromb Vasc Biol 2013; 33: 2021-2025

3 Welty F. Hypobetalipoproteinemia and abetalipoproteinemia. Curr Opin Lipidol 2014; 25: $161-168$

\section{Bibliography}

DoI http://dx.doi.org/

10.1055/s-0034-1391832

Endoscopy 2015; 47: E223-E224

(c) Georg Thieme Verlag KG

Stuttgart · New York

ISSN 0013-726X

\section{Corresponding author}

\section{Lobke Desomer, MD}

Department of Gastroenterology De Pintelaan 185

Gent

9000

Belgium

Fax: +00-32-93324984

lobkedesomer@icloud.com 